\title{
Painful Hand Swelling after a Roller Coaster Ride
}

\author{
Felicitas Stoll', Britta Heilmeier ${ }^{1,2}$, Boris Radeleff ${ }^{3}$,Hugo A Katus ${ }^{1,2}$ and Oliver J Müller ${ }^{1,2,4^{*}}$ \\ ${ }^{1}$ Department of Internal Medicine III, University of Heidelberg, Germany \\ ${ }^{2}$ DZHK (German Center for Cardiovascular Research), Partner Site Heidelberg/Mannheim, Germany \\ ${ }^{3}$ Department of Diagnostic and Interventional Radiology, University Hospital Heidelberg, Germany \\ ${ }^{4}$ Department of Internal Medicine III, University of Kiel, Germany
}

*Corresponding author: Prof. Dr. Oliver J. Müller, Department of Internal Medicine III, University of Kiel, Arnold-HellerStr. 3, 24105 Kiel, Germany, Tel: 0049-431-50022950, Fax:0049-431-50022954, E-mail: Oliver.Mueller@uksh.de

\begin{abstract}
We present a case report on the Achenbach's syndrome, a condition characterized by a painful hematoma and swelling of the palmar side of the hand and/or fingers with a sudden onset. Symptoms are typically triggered by everyday activities like carrying a bag or pressing down a door handle. Achenbach's syndrome being rather unknown, its impressive clinical presentation might unsettle both patients and physicians and entail elaborate diagnostic measures despite its in fact benign and self-limiting character.
\end{abstract}

\section{Introduction}

A patient's presentation to the out-patient clinic of the Angiology department because of acral bluish discoloration might -after the exclusion of acute, severe diagnoses - represent a challenge especially in rather young or middle-aged individuals; the visible symptoms understandably cause anxiety, in many cases however they represent a benign condition [1].

"Paroxysmal hand hematoma" (also called "Paroxymsal Finger Hematoma", or "Finger Apoplexia") was first described in 1958 by Achenbach ("Achenbach's Syndrome") [2]. It is a benign and self-limiting, but often painful bruising with a typically sudden onset after use of the respective hand.

\section{Case Report}

The 49-year-old craftsman presented himself to our Angiology department because of a painful swelling and bluish discoloration on the palmar sides of both hands. The symptoms had first occurred on the right side af- ter performing manual labour about ten months ago; thereafter, the patient preferably used the left hand, where the same symptoms occurred. Another time, the symptoms had once been provoked by a roller-coaster ride. The symptoms were self-limiting after two weeks at the most. When the patient presented himself to our department, there was no discoloration visible, however he described residual tightness in the fingers of both hands, which was improvable by cooling. The patient reported to avoid manual labour and exposure to heat in fear of provoking the symptoms again. Smoking was stopped more than ten years ago, and there were no comorbidities obviously related to the symptoms. The patient did not take any medication and had not received any treatment for his symptoms.

Before the presentation to our department, a series of MR-angiography scans had already been performed. These had shown circular angiomatous alterations along the phalanges, less along the metacarpalia of $D$ II - IV on both hands (Figure 1). The patient was then referred to our department for further evaluation of the suspected angiomatous malformation. On physical examination, the veins in the palms of both hands appeared to prominently shine through the skin; there was no swelling or neurological dysfunction. Duplex ultrasound of the arm arteries revealed a normal triphasic pattern with rather slow flows in the lower arm of 39 $\mathrm{cm} / \mathrm{sec}$ in the ulnar artery and of $32 \mathrm{~cm} / \mathrm{sec}$ in the radial artery; ultrasound of the carotid arteries and the aorta did not show any abnormalities. On the oscillography of the finger arteries, the amplitudes were slightly re-

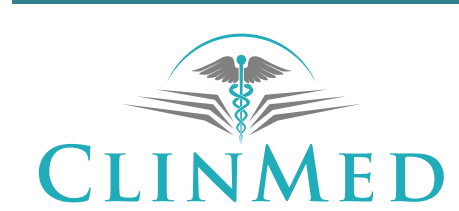

INTERNATIONAL LIBRARY

Citation: Stoll F, Heilmeier B, Radeleff B, Katus HA, Müller OJ (2018) Painful Hand Swelling after a Roller Coaster Ride. Clin Med Rev Case Rep 5:206. doi.org/10.23937/2378-3656/1410206

Accepted: March 14, 2018: Published: March 16, 2018

Copyright: (c) 2018 Stoll F, et al. This is an open-access article distributed under the terms of the Creative Commons Attribution License, which permits unrestricted use, distribution, and reproduction in any medium, provided the original author and source are credited. 


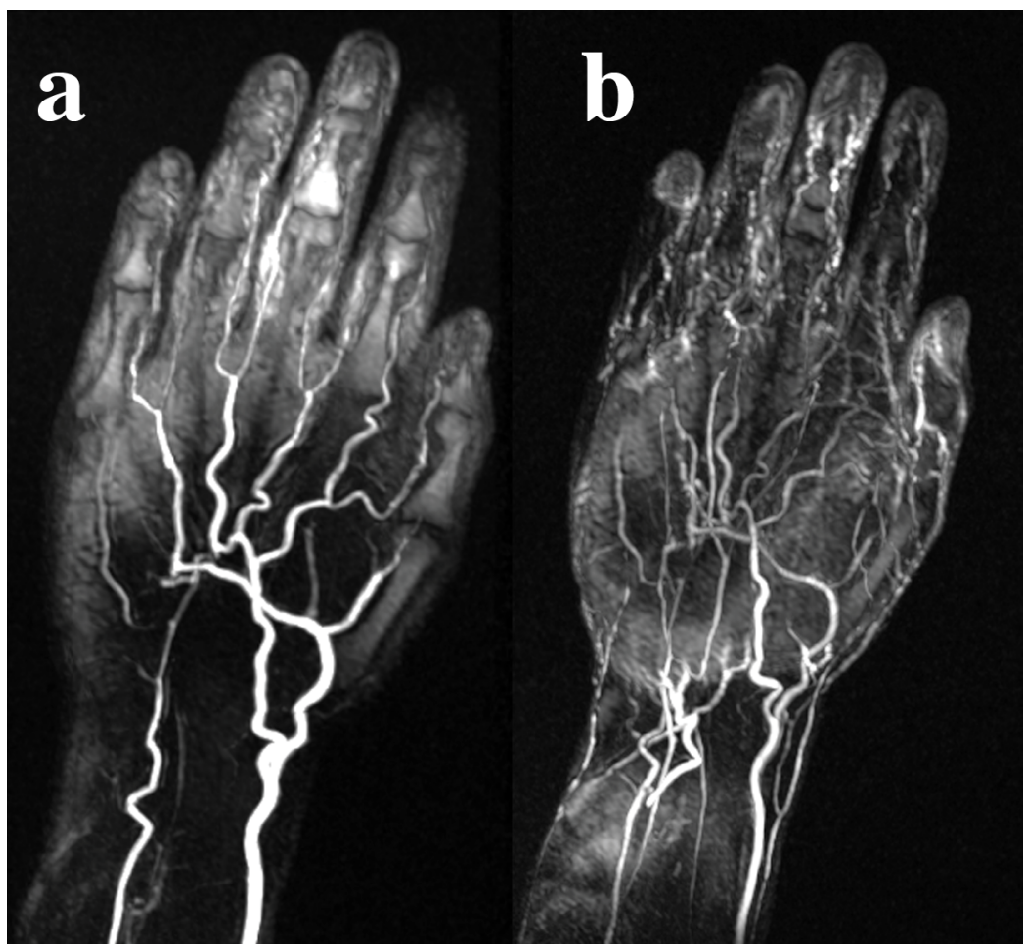

Figure 1: 3D MR-angiography showing suspected arterial angiomatous alterations alongside the acra a) Arterial and b) Venous phase.

duced. Digital subtraction angiography showed reduced blood flow in both hands, however there were no signs of an arterial malformation. To further evaluate venous blood flow, a CT-angiography was performed which did not confirm the suspected venous fistula or AV-malformation while lower arm arteries appeared rather small. Vasculitis serology revealed slightly alterated ANA-antibodies of 1:160, but was otherwise insignificant as well.

\section{Discussion}

Achenbach's syndrome is characterised by a typically painful hematoma on the palmar side of the hand and/ or fingers of sudden onset [2] with the middle and the index finger being most often affected [3]. Some authors describe a short intermediate stage of less than one hour between the onset of pain and the typical bluish discoloration during which the affected finger is pale and cold [4]. A significant swelling as well as a feeling of tension or tightness there can accompany the hematoma $[2,5]$. Symptoms may occur after everyday activities like carrying a bag, pressing down a door handle or housework $[2,4]$. For example, there has been a series of cases reported from a post office where work included tying up parcels [5]. However, there are reports indicating that often no precedent trauma can be recalled $[3,6-8]$.

Achenbach's syndrome occurs more frequently in women than in men $[5,9,10]$. Affected patients are younger than those who suffer from peripheral vascular disease, but the onset might be later than that of Raynaud's syndrome [1].

The prevalence of Achenbach's syndrome is unknown. Kordzadeh, et al. included 12 cases into their re- view of Achenbach's syndrome in 2015 [9], but missed out on the cases by Achenbach himself, Jung, Singer and Nitzschner [2,4-6] in the German-speaking literature of the 1950s and 1960s, as well as on a more recent case series from Germany by Thies, et al. [10]; with Jung [4] presenting a series of as many as 40 cases, they sum up to another 65 cases. Interestingly, there have been a few more case reports on Achenbach's syndrome in the international literature just lately, all of which are from Japan [11-13]. Altogether, around 80 cases of Achenbach's syndrome have been described in the literature since the 1950s.

Regarding the pathogenesis of the syndrome, there has been the assumption that the attacks might be associated with reduced digital blood flow [14] which might explain the sudden sensation of sharp pain. This is probably spastic [4], which would be in line with Achenbach, who thought that the phenomenon was set off by neuro-vegetative pathways: He discussed the contribution of mental stress as well as hereditary and allergic dispositions [2]. Indeed, rheography of the affected finger(s) in one study showed reduced blood flow [4,6], which significantly improved after warming, indicating underlying vasospasm [6]. Another explanation of the reduced blood flow might be compression by the hematoma [4]. In contrast to these dynamic causes of hypoperfusion, hypoplasia of the ulnar artery on the same hand side has also been described [10]. In our patient also lower arm arteries were rather small, and blood flow was reduced even outside of an attack.

Symptoms disappear within a few days [9] and may reoccur after years on the same or on the other hand 
[2]. In the meantime, patients are typically asymptomatic besides residual venectasia on the volar side of the hand and fingers, especially in the regions of the joints $[2,10]$. This is thought to be the origin of the typical hematoma [4]. The circular angiomatous alterations along the phalanges seen on the first MR scans of our patient might correlate with venectasia.

Except for history and clinical presentation, there are no established diagnostic measures; any further diagnostic efforts should therefore be rather aimed at differential diagnosis of (potentially threatening) diseases such as atherosclerosis, Takayasu's disease, Giant cell arteritis, aneurysmal disease, thoracic outlet syndrome, trauma and vibration-induced injury, Collagen vascular disease, Buerger's disease, vasospasm, microemboli, cold injury, drug-induced conditions and haematological diseases (e.g. polycythaemia). Another condition associated with similar symptoms, but also involving other parts of the body is the Painful Bruising Syndrome (autoerythrocyte sensitisation syndrome, Gardner-Diamond syndrome) that is often associated with psychiatric comorbidities [15]. Some point out that while in ischemia the fingertip is affected, it is not in Achenbach's syndrome $[6,15]$. In a case study of 22 patients presenting themselves with blue discoloration of fingers, 17 patients were asymptomatic at a 6-week follow-up (most of them being on antiplatelet therapy) [1]. The authors come to the conclusion that, if there is no systemic disease underlying, "patients can be reassured that the condition should resolve spontaneously and that further episodes are unlikely" [1]. Regarding the benign nature of Achenbach's syndrome, the value of invasive tests can be disputed [15]. However, pathologic findings in the non-invasive tests might necessitate the ruling out of other conditions as mentioned above.

In Achenbach's syndrome, the patient can be advised to avoid use of the affected hand as long as symptoms are present [4]. Achenbach also saw the consulted physician's main task in reassuring the patient of the syndrome's harmlessness [2], even if episodes might reoccur. In cases, where the syndrome tends to frequently reoccur, treatment of the digital venectasia has been suggested in literature [4], which, however, has - to our knowledge - never been translated into practice, most likely due to the benign and self-limiting character of the syndrome.

\section{Conclusion}

While the pathogenesis of Achenbach's syndrome has been subject to discussion, there is a consensus on its benign nature. Diagnostic measures should be aimed at ruling out (potentially threatening) differential diagnoses and if so, the patient can be reassured of the benign nature of the syndrome. The case report presented here shows a typical example of Achenbach's syndrome: It illustrates the challenges in diagnostics, even the more in modern medicine, and contributes to the limited amount of literature not only on the suspected pathogenesis of this condition.

\section{Conflict of Interest Disclosure}

The authors declare that there is no conflict of interest regarding the publication of this paper.

\section{References}

1. Cowen R, Richards T, Dharmadasa A, Handa A, Perkins JMT (2008) The acute blue finger: Management and outcome. Ann R Coll Surg Engl 90: 557-560.

2. Achenbach $W$ (1958) Paroxysmal hematoma of the hand. Medizinische 52: 2138-2140.

3. Eikenboom JC, Cannegieter SC, Briët E (1991) Paroxysmal finger haematoma: A neglected syndrome. Thromb Haemost 66: 266.

4. Jung EG (1964) Paroxysmal Hematoma of the Fingers. Schweiz Med Wochenschr 94: 458-460.

5. Nitzschner H (1967) On the etiology of Achenbach's syndrome. Z Haut Geschlechtskr 42: 141-144.

6. Singer R (1962) On the symptoms and diagnosis of finger apoplexy (paroxysmal hematoma of the hand). Wien Klin Wochenschr 74: 741-743.

7. Frerix M, Richter K, Müller-Ladner U, Hermann W (2015) Achenbach's syndrome (paroxysmal finger hematoma) with capillaroscopic evidence of microhemorrhages. Arthritis Rheumatol 67: 1073.

8. Harper CM, Waters PM (2013) Acute idiopathic blue finger: Case report. J Hand Surg Am 38: 1980-1982.

9. Kordzadeh A, Caine PL, Jonas A, Rhodes KM, Panayiotopolous YP (2016) Is Achenbach's syndrome a surgical emergency? A systematic review. Eur J Trauma Emerg Surg 42: 439-443.

10. Thies K, Beschorner U, Noory E, Zeller T (2012) Achenbach's syndrome revisited. Vasa 41: 366-370.

11. Yamamoto Y, Yamamoto S (2017) Achenbach's Syndrome. N Engl J Med 376: e53.

12. Yamada T (2018) Achenbach's syndrome in an elderly woman. J Gen Fam Med 19: 65-66.

13. Takeuchi H, Uchida HA, Okuyama Y, Wada J (2016) Acute idiopathic blue fingers: A young man with Achenbach's syndrome. BMJ Case Rep 2016: 10.

14. Robertson A, Liddington MI, Kay SP (2002) Paroxysmal finger haematomas (Achenbach's syndrome) with angiographic abnormalities. J Hand Surg Br 27: 391-393.

15. Khaira HS, Rittoo D, Vohra RK, Smith SR (2001) The non-ischaemic blue finger. Ann R Coll Surg Engl 83: 154157. 